\title{
Lo scambio eritrocitario
}

\author{
Tiziana Tison, Piero Marson, Giustina De Silvestro
}

Servizio di Aferesi Terapeutica, Unità Operativa Complessa Immunotrasfusionale, Azienda Ospedaliera di Padova, Padova

\begin{abstract}
The ERITRO-EXChange
Abstract. Red blood cell (RBC) exchange transfusion is a procedure in which a machine removes the patient's abnormal RBCs using a centrifuge to separate the blood into its various parts. These abnormal cells are replaced with RBC units from volunteer healthy blood donors. RBC exchange transfusion can be done either using needles placed in the peripheral veins of each arm or using a central venous catheter. Since this technique is often performed in pediatric patients, it is important to evaluate its indications, and it is also necessary to consider some particular aspects for the apheretic procedure. RBC exchange transfusion is used to treat serious complications of sickle-cell disease, including stroke and acute chest syndrome, and can also be used in certain infections such as malaria or babesiosis, in which red blood cells are damaged.
\end{abstract}

Key words: Red-blood cell exchange transfusion, Vascular access, Transfusion complications, Sickle-cell disease

Conflict of interest: None.

Financial support: None.

Ricevuto: 18 Gennaio 2013; Accettato: 27 Febbraio 2013

L'eritroexchange (EEx), o scambio eritrocitario, è una procedura che consiste nella rimozione, mediante un apparecchio denominato separatore cellulare, dei globuli rossi patologici del paziente, per emoglobinopatia $(1,2)$ o infezione parassitaria, che vengono sostituiti con globuli rossi di un donatore.

La procedura si effettua secondo la seguente modalità: il sangue del paziente prelevato tramite accesso venoso viene convogliato, attraverso un kit sterile monouso preliminarmente applicato al separatore, a una camera di centrifugazione. La centrifugazione consente di separare le varie componenti del sangue in base al loro peso specifico, stratificandole. Attraverso la lettura di una scala colorimetrica è possibile selezionare lo "strato" che si vuole rimuovere, quello, cioè, che, nel caso dell'EEx, contiene i globuli rossi patologici. Un sistema di pompe peristaltiche invia le cellule patologiche a una sacca di scarto; vengono sostituite con una pari quantità di sangue omologo e restituito al paziente attraverso un accesso vascolare.

Le unità di emazie preparate dal laboratorio trasfusionale sono unità fresche, possibilmente raccolte da non più di 15 gior$\mathrm{ni}$, isogruppo, isoassetto per il sistema $\mathrm{Rh}$ e l'antigene Kell e deleucocitate. Una volta eseguite le prove di compatibilità, poche ore prima di essere utilizzate, le unità vengono, nella maggior parte dei casi, private, mediante centrifugazione, del SAG-mannitolo, abitualmente presente nella sacca di eritrociti con la funzione di conservante, poiché tale sostanza può risultare nefrotossica nei bambini e nei soggetti con insufficienza renale. Per mantenere invariato l'ematocrito, si sostiuisce il SAG-mannitolo con della soluzione fisiologica in pari volume. Il volume solitamente scambiato è pari a $25-30 \mathrm{~mL} / \mathrm{kg}$.

\section{Complicanze}

Nei soggetti politrasfusi può accadere che si verifichi una risposta immunitaria nei confronti di antigeni minori presenti sulla superficie degli eritrociti. In questo caso, è indispensabile ricercare unità di eritrociti che non presentino sulla loro superficie lo specifico antigene verso cui il paziente si è immunizzato. Talora, questa ricerca può risultare alquanto complessa, soprattutto quando ci si trova di fronte a fenotipi eritrocitari rari nelle popolazioni caucasiche, dato che il bacino dei donatori è rappresentato nella grande maggioranza da donatori di tale origine, mentre i pazienti provengono prevalentemente da paesi africani.

Per quanto riguarda la problematica infettivologica correlata alla trasfusione di numerose unità di emazie, è possibile affermare che, nel corso dell'ultimo decennio, la sicurezza trasfusionale ha raggiunto livelli tali nel settore della prevenzione delle malattie trasmissibili che tale aspetto è ormai in secondo piano rispetto ad altre possibili complicanze correlate alla trasfusione. Tuttavia, non viene sottovalutato l'aspetto dei patogeni emergenti, per cui esiste, nel sistema trasfusionale italiano, un attivo sistema di emovigilanza.

Contrariamente alla semplice terapia trasfusionale, l'EEx consente di ottenere un bilancio marziale negativo e, pertanto, è un approccio terapeutico da considerare in caso di emocromatosi secondaria clinicamente significativa, frequente soprattutto in soggetti con patologie croniche (come la drepanocitosi). Durante tutta la procedura, il sangue che entra nel separatore cellulare non deve coagulare e, pertanto, viene infuso continua- 
mente un anticoagulante a base di citrato (ACD-A), che ha la funzione di legare il calcio, cofattore della cascata coagulativa. Possono, pertanto, comparire sintomi di ipocalcemia, quali formicolii alle mani o al volto, lievi contrazioni muscolari e senso di nausea. Tali disturbi possono essere rapidamente corretti con la somministrazione di calcio per via endovenosa, che, solitamente, viene infuso nel corso di tutta la procedura tramite una pompa, al fine di prevenire la comparsa di tali disturbi.

\section{Criticità}

L'esecuzione dell'EEx richiede la disponibilità di accessi venosi che possano garantire un flusso adeguato, che non siano troppo invasivi e che siano semplici da reperire e poco costosi, confortevoli e psicologicamente accettabili da parte del paziente $(3,4)$. Purtroppo, non sempre sono reperibili accessi adeguati e, pertanto, nelle situazioni in cui la procedura di EEx assume le caratteristiche di urgenza non demandabile, è necessario il posizionamento di un catetere venoso centrale (CVC). La scelta del tipo di CVC deve tenere presente $\mathrm{i}$ seguenti aspetti: il tipo di uso, la frequenza di utilizzo e il tempo di permanenza previsto.

Spesso, la procedura di EEx viene effettuata su pazienti pediatrici, elemento che aggiunge la necessità di gestire alcuni aspetti procedurali che possono diventare critici. Nei pazienti pediatrici diviene obbligatorio ricorrere al posizionamento di un CVC non solo per la necessità di ottenere un flusso adeguato, ma anche per la mancanza di compliance del paziente. Le Linee Guida del trattamento della drepanocitosi (5) prevedono il trattamento profilattico dei soggetti con caratteristiche cliniche a elevato rischio, che verranno elencate in seguito; non sempre è possibile attuare tale indicazione, poiché mancano accessi venosi periferici idonei e diviene difficile proporre il posizionamento di un CVC a due vie che richieda una lunga permanenza e una manutenzione domiciliare e che comporti rischi di eventi trombotici. È da poco entrato in commercio un catetere tipo Port (Smart-Port) che consente flussi idonei per la procedura aferetica e che presenta caratteristiche di maggiore maneggevolezza e tollerabilità; tale dispositivo potrebbe cambiare l'approccio nei confronti dei pazienti pediatrici ad alto rischio, ma che non presentano ancora una "maturità" adeguata del patrimonio venoso.

Anche il peso del paziente pediatrico deve essere tenuto in adeguata considerazione. Infatti, è previsto un volume di circolazione extracorporea di circa 150-200 mL che, in soggetti a basso peso, può creare un rischio di shock ipovolemico. Tale criticità deve essere corretta tramite il riempimento preliminare del circuito con emazie deleucocitate, se il peso è inferiore ai $15 \mathrm{~kg}$, o con una soluzione di fisiologica e albumina al $4 \%$ per un peso compreso tra i $15 \mathrm{~kg}$ e i $25 \mathrm{~kg}$.

\section{Indicazioni terapeutiche}

Le malattie per le quali il trattamento di EEx può essere indicato sono, secondo i criteri dell'American Society For Apheresis (ASFA) pubblicati nel 2010 (6), quelle riportate nella seguente Tabella I.
TABELLA I

\begin{tabular}{l} 
Patologia \\
Categoria di trattamento \\
\hline Malaria severa \\
II \\
Babesiosi severa \\
I \\
Drepanocitosi (ictus in fase acuta) \\
I \\
Drepanocitosi (profilassi dell'ictus) \\
II \\
Drepanocitosi (prevenzione accumulo ferro) \\
II \\
Drepanocitosi (acute chest syndrome) \\
II \\
Drepanocitosi (insufficienza multiorgano) \\
III
\end{tabular}

Nel caso della malaria e della babesiosi, l'EEx ha lo scopo di ridurre rapidamente il livello della parassitemia che danneggia i globuli rossi e, contemporaneamente, limita il rischio di danni determinati da detriti cellulari e tossine derivate dal parassita, che possono innescare una CID o un'insufficienza renale. Criteri clinici per l'esecuzione dell'aferesi sono:

- parassitemia $>10 \%$

- quadro clinico di coinvolgimento multiorgano e, in particolare, per la malaria, presenza di un interessamento cerebrale. Più articolate sono le indicazioni per l'EEx nel caso della drepanocitosi, che possono essere distinte in acute e preventive. - Indicazioni al trattamento in acuto

È ampiamente riconosciuto in letteratura l'intervento dell'aferesi in urgenza, cioè il prima possibile, nel paziente drepanocitico, nel caso si verifichi uno stroke, in corso di acute chest syndrome con importante compromissione respiratoria, come preparazione a un intervento di chirurgia maggiore od oftalmica (generalmente programmabili), in caso di un'insufficienza multiorgano e in previsione di esami con l'utilizzo di mezzi di contrasto ad alte dosi. Sono ancora discusse e controverse le indicazioni in caso di crisi dolorose severe non responsive al trattamento medico standard e in caso di priapismo.

- Indicazioni al trattamento preventivo

Soggetti in età pediatrica che presentino fattori di rischio che predispongono allo sviluppo di complicanze neurologiche o che abbiano già sofferto di danni neurologici trovano beneficio nel trattamento di eritroexchange programmato ogni 4-6 settimane per prevenire nuovi eventi. Altre indicazioni sono: l'intolleranza al trattamento con idrossiurea, l'intolleranza alla ferrochelazione, l'emocromatosi post-trasfusionale, la preparazione a interventi chirurgici e il riscontro di un flusso elevato al Doppler transcranico e nel corso della gravidanza. L'obietti- 
vo, nel corso del trattamento preventivo, è quello di mantenere o di portare i livelli di emoglobina $\mathrm{S}$ al di sotto del $30 \%$.

Le Linee Guida Pubblicate dall'Associazione Italiana Ematologia Oncologia Pediatrica (AIEOP) (7) nel 2011 identificano i criteri di eleggibilità al trapianto allogenico di cellule staminali ematopoietiche per i soggetti affetti da drepanocitosi. Prima dell'inizio del regime di condizionamento, è indicata l'esecuzione di una procedura di EEx, in modo da portare il paziente al trapianto nella migliore condizione possibile.

\section{Riassunto}

Lo scambio eritrocitario è una procedura in cui, utilizzando un separatore cellulare, si rimuovono i globuli rossi anomali del paziente, che vengono rimpiazzati con globuli rossi di un donatore volontario. Lo scambio eritrocitario può essere effettuato sia utilizzando accessi venosi periferici che un catetere venoso centrale. Dal momento che spesso si tratta di pazienti pediatrici è importante valutare attentamente le indicazioni ed è necessario considerare alcuni aspetti particolari della procedura aferetica. Lo scambio eritrocitario è indicato in caso di complicanze severe della drepanocitosi, quali l'ictus e l'acute chest syndrome, e nel caso di alcune infezioni quali la malaria e la babesiosi, in cui si verifica, ad opera dei parassiti, un danno eritrocitario.
Parole chiave: Scambio eritrocitario, Accessi vascolari, Complicanze trasfusionali, Drepanocitosi

Dichiarazione di conflitto di interessi: Gli Autori dichiarano di non avere conflitto di interessi.

Contributi economici degli autori: Gli Autori dichiarano di non aver ricevuto sponsorizzazioni economiche per la preparazione dell'articolo.
Indirizzo degli Autori:

Dr.ssa Tiziana Tison

U.O.C. Immunotrasfusionale

Azienda Ospedaliera di Padova

Via Giustiniani 2

35128 Padova

tiziana.tison@sanita.padova.it

\section{Bibliografia}

1. Swerdlow PS. Red cell exchange in sickle cell disease. Hematology Am Soc Hematol Educ Program 2006; 48-53.

2. Whal S, Quirolo KC. Current issues in blood transfusion for sickle cell disease. Curr Opin Pediatr 2009; 21: 15-21.

3. De Silvestro G, Bagatella P, Vicarioto M, et al. The Italian SIdEM registry for apheresis: an overview of the 2005 statistics. Int J Artif Organs 2008; 31: 354-62.

4. De Silvestro G, Tison T, Vicarioto M, et al. The Italian Registry of Pediatric Therapeutic Apheresis: a report on activity during 2005. J Clin Apher 2009; 24: 1-5.
5. Roseff SD. Sickle cell disease: a review. Immunohematology 2009; 25: 67-74.

6. Szczepiorkowski ZM, Winters JL, Bandarenko N, et al. Guidelines on the use of therapeutic apheresis in clinical practice -- evidence-based approach from the Apheresis Apllications Committee of the American Society for Apheresis. J Clin Apher 2010; 25: 83-117.

7. Associazione Italiana Ematologia Oncologia Pediatrica (AIEOP). Gruppo di Lavoro "Patologia del globulo rosso". Raccomandazioni per la gestione della malattia drepanocitica in età pediatrica in Italia. 2010. 